\title{
Understanding Li-O2 Battery Cathode Product Formation via Liquid Cell Electron Microscopy
}

Saran Pidaparthy ${ }^{1}$, Chang Liu ${ }^{1}$, Ryan Stephens ${ }^{2}$, Qian Chen ${ }^{1}$ and Jian-Min Zuo ${ }^{1}$

${ }^{1}$ University of Illinois at Urbana-Champaign, Urbana, Illinois, United States, ${ }^{2}$ Shell International Exploration and Production Inc., Houston, Texas, United States

The lithium-oxygen $\left(\mathrm{Li}-\mathrm{O}_{2}\right)$ battery is a beyond lithium-ion (Li-ion) technology that can provide a theoretical energy density of $3500 \mathrm{Wh} / \mathrm{kg}$, which is $\sim 10$ times greater than what state-of-the-art Li-ion batteries can deliver [1]. Despite the energy benefit of $\mathrm{Li}-\mathrm{O}_{2}$ batteries, there are significant scientific challenges to overcome to enable this technology. In particular, we need a deeper understanding of the cathode product evolution during battery cycling and its impact on the performance of the battery [1]-[3]. While there have been many efforts in elucidating $\mathrm{Li}-\mathrm{O}_{2}$ cathode product evolution during battery cycling, there is still disagreement on the growth mechanisms responsible limiting the battery efficiency. With the advent of liquid cell electron microscopy and electrochemical biasing sample holders, such transient battery phenomena can be measured at high spatial and temporal resolution. When this technique is adapted to a $\mathrm{Li}-\mathrm{O}_{2}$ system, we have a method to resolve the growth mechanism by capturing the discharge product growth processes on a $\mathrm{Li}-\mathrm{O}_{2}$ cathode in real-time during battery cycling.

Here, we report direct observation of $\mathrm{Li}-\mathrm{O}_{2}$ battery cathode discharge product formation via in-situ liquid cell electron microscopy. The experiments were carried out using a Hitachi 9500 environmental TEM with a LaB6 emitter (Hitachi High-Technologies Corp.) operated at $200 \mathrm{kV}$ combined with a liquid-flow, electrochemical-biasing TEM holder (Hummingbird Scientific). To mitigate the electron beam effect, the dose rate was maintained at $<0.7 \mathrm{e}^{-} / \AA^{2}$ s. Adapted from prior literature [4]-[6], the Li-O 2 micro battery was assembled using commercial electrochemical biasing chips (Hummingbird Scientific) with a gold working electrode and lithium iron phosphate $(\mathrm{LiFePO} 4)$ counter electrode. During image acquisition, we flowed an oxygen-saturated electrolyte of $1.0 \mathrm{M}$ lithium perchlorate $\left(\mathrm{LiClO}_{4}\right)$ in dimethyl sulfoxide (DMSO) through the holder's liquid channels and simultaneously performed galvanostatic discharging at $1000 \mathrm{nA}$.

During discharging, we observe surface growth of discharge products on the working electrode in realtime (see Figure 1). Following an initial incubation period, which is likely due to a nucleation overpotential, these particles grow via kinetic roughening on the working electrode surface [7]. The discharge products appear as round deposits across the entire surface with an average radius of $50 \mathrm{~nm}-$ $70 \mathrm{~nm}$. At higher magnifications, the surface of these particles appears as dense, fractal deposits. Postmortem SEM also shows evidence of toroidal morphology, which is consistent electrochemically formed crystalline $\mathrm{Li}_{2} \mathrm{O}_{2}$ [1], [8]. From electron diffraction, the discharge products were identified as a nanocrystalline combination of both lithium peroxide $\left(\mathrm{Li}_{2} \mathrm{O}_{2}\right)$ and lithium hydroxide $(\mathrm{LiOH})$ phases. We further benchmarked these results against a lab-scale $\mathrm{Li}-\mathrm{O}_{2}$ cell with a similar configuration as the liquid cell setup. We characterized the lab-scale cathode after discharging via SEM, XRD, and TEM, and found similar product morphology, phases, and microstructure [9]. 

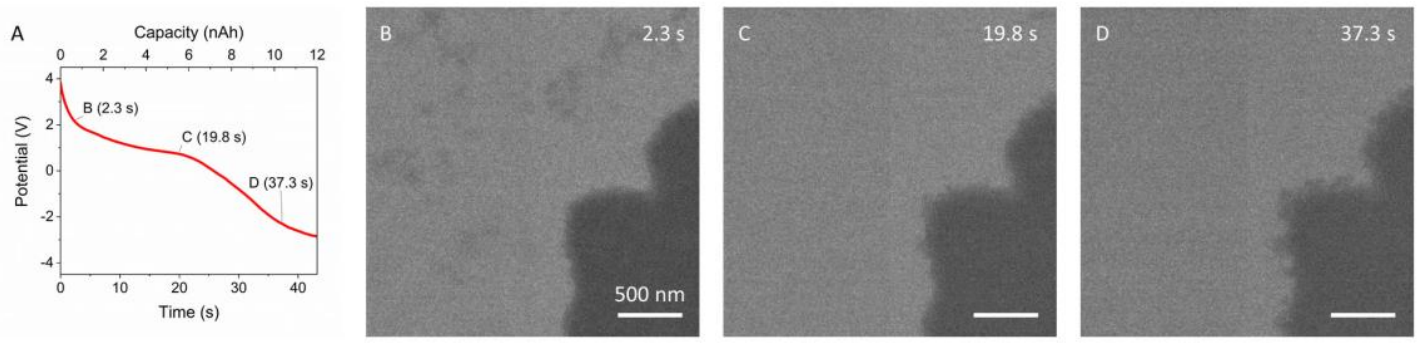

Figure 1. A correlation of galvanostatic discharging data with a time-series of product growth on the LiO2 micro battery working electrode. (A) Shows the acquired discharging curve with highlighted time stamps at (B) $2.3 \mathrm{~s}$, (C) $19.8 \mathrm{~s}$, and (D) $37.3 \mathrm{~s}$. (B)-(D) Shows the corresponding frames from a time-series image sequence of the growth of electrochemically formed discharge products on the working electrode surface. Scale bar: $500 \mathrm{~nm}$.

\section{References}

[1] J. Lu, et al., "Aprotic and Aqueous Li-O2 Batteries," Chem. Rev., vol. 114, no. 11, pp. 5611-5640, Jun. 2014.

[2] Z. Ma et al., "A review of cathode materials and structures for rechargeable lithium-air batteries," Energy Environ. Sci., vol. 8, no. 8, pp. 2144-2198, 2015.

[3] L. Grande et al., "The Lithium/Air Battery: Still an Emerging System or a Practical Reality?," $A d v$. Mater., vol. 27, no. 5, pp. 784-800, Feb. 2015.

[4] C. Yang et al., "Direct Observations of the Formation and Redox-Mediator-Assisted Decomposition of $\mathrm{Li}_{2} \mathrm{O}_{2}$ in a Liquid-Cell $\mathrm{Li}-\mathrm{O}_{2}$ Microbattery by Scanning Transmission Electron Microscopy," Adv. Mater., vol. 29, no. 41, p. 1702752, Nov. 2017.

[5] C. Hou et al., "Operando observations of $\mathrm{RuO}_{2}$ catalyzed $\mathrm{Li}_{2} \mathrm{O}_{2}$ formation and decomposition in a $\mathrm{Li}$ O micro-battery," Nano Energy, vol. 47, pp. 427-433, 2018.

[6] P. Liu et al., "Operando characterization of cathodic reactions in a liquid-state lithium-oxygen microbattery by scanning transmission electron microscopy," Sci. Rep., vol. 8, no. 1, p. 3134, 2018.

[7] N. M. Schneider, et al., "Nanoscale evolution of interface morphology during electrodeposition," Nat. Commun., vol. 8, no. 1, p. 2174, 2017.

[8] R. R. Mitchell, et al., "Mechanisms of Morphological Evolution of $\mathrm{Li}_{2} \mathrm{O}_{2}$ Particles during Electrochemical Growth," J. Phys. Chem. Lett., vol. 4, no. 7, pp. 1060-1064, Apr. 2013.

[9] Funding for this work was provided by the Energy \& Biosciences Institute through the EBI-Shell program. 